\title{
Twelve-month results of a multicenter, blinded, pilot study of a novel peptide (B2A) in promoting lumbar spine fusion
}

\author{
Zeeshan Sardar, MD, CM, MSc, ${ }^{1}$ David Alexander, MD, ${ }^{2}$ William Oxner, MD, ${ }^{2}$ \\ Stephan du Plessis, MD, ${ }^{3}$ Albert Yee, MD, ${ }^{4}$ Eugene K. Wai, MD, MSc, ${ }^{5}$ D. Greg Anderson, MD, ${ }^{6}$ \\ and Peter Jarzem, MD'
}

\begin{abstract}
${ }^{1}$ McGill University Health Centre, McGill University, Montreal, Quebec; ${ }^{2}$ Queen Elizabeth II Health Sciences Centre, Dalhousie University, Halifax, Nova Scotia; ${ }^{3}$ Foothills Medical Center, University of Calgary, Alberta; ${ }^{4}$ Sunnybrook Health Sciences Centre, University of Toronto, Ontario; ${ }^{5}$ Ottawa Hospital, University of Ottawa, Ontario, Canada; and ${ }^{6}$ Thomas Jefferson Hospital, Philadelphia, Pennsylvania
\end{abstract}

OBJECT Failure of fusion after a transforaminal lumbar interbody fusion (TLIF) procedure is a challenging problem that can lead to ongoing low-back pain, dependence on pain medication, and inability to return to work. B2A is a synthetic peptide that has proven efficacy in achieving fusion in animal models and may have a better safety profile than bone morphogenetic protein. The authors undertook this study to evaluate the safety and efficacy of B2A peptide-enhanced ceramic granules (Prefix) in comparison with autogenous iliac crest bone graft (ICBG, control) in patients undergoing single-level TLIF.

METHODS Twenty-four patients with single-level degenerative disorders of the lumbar spine at L2-S1 requiring TLIF were enrolled between 2009 and 2010. They were randomly assigned to 3 groups: a control group (treated with ICBG, $n$ = 9), a Prefix 150 group (treated with Prefix $150 \mu \mathrm{g} / \mathrm{cm}^{3}$ granules, $n=8$ ), and a Prefix 750 group (treated with Prefix 750 $\mu \mathrm{g} / \mathrm{cm}^{3}$ granules, $n=7$ ). Outcome measures included the Oswestry Disability Index (ODI), visual analog pain scale, and radiographic fusion as assessed by CT and dynamic flexion/extension lumbar plain radiographs.

RESULTS At 12 months after surgery, the radiographic fusion rate was 100\% in the Prefix 750 group, $78 \%$ in the control group, and $50 \%$ in the Prefix 150 group, although the difference was not statistically significant $(p=0.08)$. At 6 weeks the mean ODI score was 41.0 for the control group, 27.7 for the Prefix 750 group, and 32.2 for the Prefix 150 group, whereas at 12 months the mean ODI was 24.4 for control, 31.1 for Prefix 750, and 29.7 for Prefix 150 groups. Complications were evenly distributed among the groups.

CONCLUSIONS Prefix appears to provide a safe alternative to autogenous ICBG. Prefix 750 appears to show superior radiographic fusion when compared with autograft at 12 months after TLIF, although no statistically significant difference was demonstrated in this small study. Prefix and control groups both appeared to demonstrate comparable improvements to ODI at 12 months.

Clinical trial registration no.: NCT00798902 and NCT00798239 (clinicaltrials.gov)

http://thejns.org/doi/abs/10.3171/2013.11.SPINE121106

KEY WORDS TLIF; spine fusion; BMP; Prefix; bone graft; degenerative disc disease; spondylolisthesis; lumbar

$\mathrm{S}$ INGLE-LEVEL transforaminal lumbar interbody fusion (TLIF) is a commonly performed procedure used to treat degenerative conditions of the lumbar spine. During this procedure the intervertebral disc is removed and a spacer is inserted between the 2 vertebral bodies to achieve interbody fusion. Failure of fusion is a challenging problem that can lead to ongoing low-back pain and dependence on pain medication and adversely impact the

ABBREVIATIONS ICBG = iliac crest bone graft; ODI = Oswestry Disability Index; PEEK = polyetheretherketone; rhBMP-2 = recombinant human bone morphogenetic protein-2; TLIF = transforaminal lumbar interbody fusion; VAS = visual analog scale.

SUBMITTED December 4, 2012. ACCEPTED November 24, 2013.

INCLUDE WHEN CITING Published online January 23, 2015; DOI: 10.3171/2013.11.SPINE121106.

DISCLOSURE This study was funded by Biosurface Engineering Technology (BioSET). Dr. Anderson reports that he is the medical monitor for the study described in this article and has received BioSET stock options for his work as medical monitor. Dr. Jarzem reports receiving support from BioSET for the study described. 
ability to return to work. The historic gold standard for graft, iliac crest bone graft (ICBG), used to fill the intervertebral disc space during spinal fusions due to its osteoinductive and osteoconductive properties as well as low immunogenicity, ${ }^{3,4,14,19-21,26,27}$ is associated with increased morbidity related to the donor site from which the graft is harvested. . $7,12-14,19,20,22,23,25,27,30,31$ These complications include nerve injury, hematoma, infection, fracture, and hernias..$^{5,13,14,20,22,23,25,30,31}$ Minimally invasive techniques ${ }^{19}$ for ICBG harvesting have been described that result in less morbidity than the regular open technique but limit the amount of cancellous bone graft that can be harvested. Even if one chose to overlook the morbidity associated with ICBG, there are instances when it is not possible to harvest bone from the iliac crest $^{3,21,27}$ due to previous harvesting or pelvic dysmorphism. ${ }^{21}$

Therefore, other biological and synthetic sources of graft have been identified. ${ }^{1-4,12-16,19-21,26,28}$ Local bone autograft from the site of surgery is commonly used in posterior lumbar interbody fusions ${ }^{17}$ and has been shown to be comparable to ICBG in achieving fusion. Several other sites for autogenous bone graft harvesting from extremities of the patients, ${ }^{20}$ including intramedullary reaming debris collected from the patient's femur, ${ }^{7,21}$ have been used in spine surgery but have not been routinely adopted due to potential complications. Nevertheless, autograft is still associated with a significant pseudarthrosis rate that may be as high as $26 \%$ in some series. ${ }^{14}$

In an effort to avoid the problems associated with autograft and the possibility of disease transmission associated with allograft, synthetic alternatives such as recombinant human bone morphogenetic protein-2 (rhBMP-2) ${ }^{9,10,29,31}$ have been developed. Unfortunately, significant complications, including some life-threatening events, have been reported with the use of rhBMP-2,2,3,10,14,24,29 The literature regarding complications with rhBMP-2 usage is quite variable, with some authors suggesting complication rates between $10 \%$ and $50 \%$ for posterior lumbar interbody fusion. Others, such as Glassman et al., ${ }^{10}$ have reported a low complication rate of $0.6 \%$ with rhBMP-2 when used for posterolateral spine fusion. This degree of variability suggests the need to evaluate the safety profile of rhBMP- 2 in a site- and procedure-specific manner ${ }^{29}$ Reported complications include radiculitis, ectopic bone formation, osteolysis, greater apparent risk of malignancy, neurological compromise from bony overgrowth, and inferior overall outcomes. ${ }^{3}$

Concerns regarding the safety of rhBMP-2 have led to interest in a number of alternatives to rhBMP-2, such as bioactive glass,,$^{12}$ recombinant platelet-derived growth factor, ${ }^{6,27}$ and platelet-rich plasma added to ICBG.$^{28}$ However, their efficacy in achieving lumbar interbody fusion in humans has not been proven.

B2A is a synthetic peptide that amplifies the biological response to native BMP-2 through interaction with BMP receptors, leading to increased osteoblast differentiation. ${ }^{18}$ As B2A requires native endogenous BMP-2 for activity, it will not form bone in areas where BMP-2 is not already present and may therefore have a better safety profile compared with exogenous rhBMPs. Potential advantages over rhBMP-2 include a reduced risk of malignancy, ectopic bone formation, and neurological compromise from bony overgrowth. However, these are theoretical advantages that have not been confirmed. B2A has both osteoinductive and osteoconductive properties and has proven superiority to autograft in achieving spinal interbody fusion in animal models, ${ }^{4,26}$ with no reports of heterotopic ossification. It has also been shown to provide accelerated bone repair in rabbit long bone defects ${ }^{18}$ and has been shown to be comparable to autograft in a pilot study assessing ankle and hindfoot arthrodesis in humans. ${ }^{11}$

Encouraged by these results, we designed the current study to test the safety and effectiveness of B2A-coated ceramic granules (Prefix) in humans. This is the first multicenter, prospective, randomized, partially blinded control trial evaluating the safety and the potential effectiveness of Prefix in comparison with autogenous ICBG (control) in achieving fusion in patients undergoing singlelevel TLIF for degenerate disorders of the lumbar spine. We report on the Canadian patients from a Phase I clinical trial, and hence the focus of this study is to establish the safety and appropriate dosage of Prefix required to achieve fusion. We hypothesize that Prefix would achieve a fusion rate equivalent or superior to that of ICBG and would be safe to use.

\section{Methods}

This study was approved by the institutional review boards of all the centers involved and conducted under Health Canada investigational testing authorization. It was registered with the ClinicalTrials.gov database (http:// clinicaltrials.gov), under the following registration numbers: NCT00798902 and NCT00798239. Twenty-four patients with single-level degenerative disorders of the lumbar spine (degenerative disc disease, spinal stenosis, spondylolisthesis) at L2-S1 requiring TLIF were enrolled between 2009 and 2010 at 5 Canadian centers (Fig. 1). Patients were recruited into the study and provided informed consent prior to randomization and assignment to the 3 treatment groups: control (ICBG), Prefix 150 (ceramic granules coated with $\mathrm{B} 2 \mathrm{~A}$ at $150 \mu \mathrm{g} / \mathrm{cm}^{3}$ granules), and Prefix 750 (ceramic granules coated with B2A at $750 \mu \mathrm{g} /$ $\mathrm{cm}^{3}$ granules). Patients were randomized (Table 1) in sequential chronological order using a block randomization protocol stratified across all of the study sites. The patients who were assigned to one of the two Prefix groups were blinded to dosage (i.e, were not aware of whether they had been assigned to the Prefix 150 or Prefix 750 group), but patients could not be blinded to control versus Prefix group assignment due to the incision necessary for harvesting the graft in patients in the control group. The patients were required to meet all inclusion and exclusion criteria as listed in Table 2.

\section{Surgical Technique and Outcome Measures}

Prefix was prepared from vials of lyophilized B2A peptide and porous, ceramic granules $(80 \%$ tri-calciumphosphate $/ 20 \%$ hydroxyapatite, Biomatlante). For the Prefix 150 group, ceramic granules were coated with B2A at $150 \mu \mathrm{g} / \mathrm{cm}^{3}$ of granules. For Prefix 750, ceramic granules were coated with B2A at $750 \mu \mathrm{g} / \mathrm{cm}^{3}$ of granules. Five cu- 


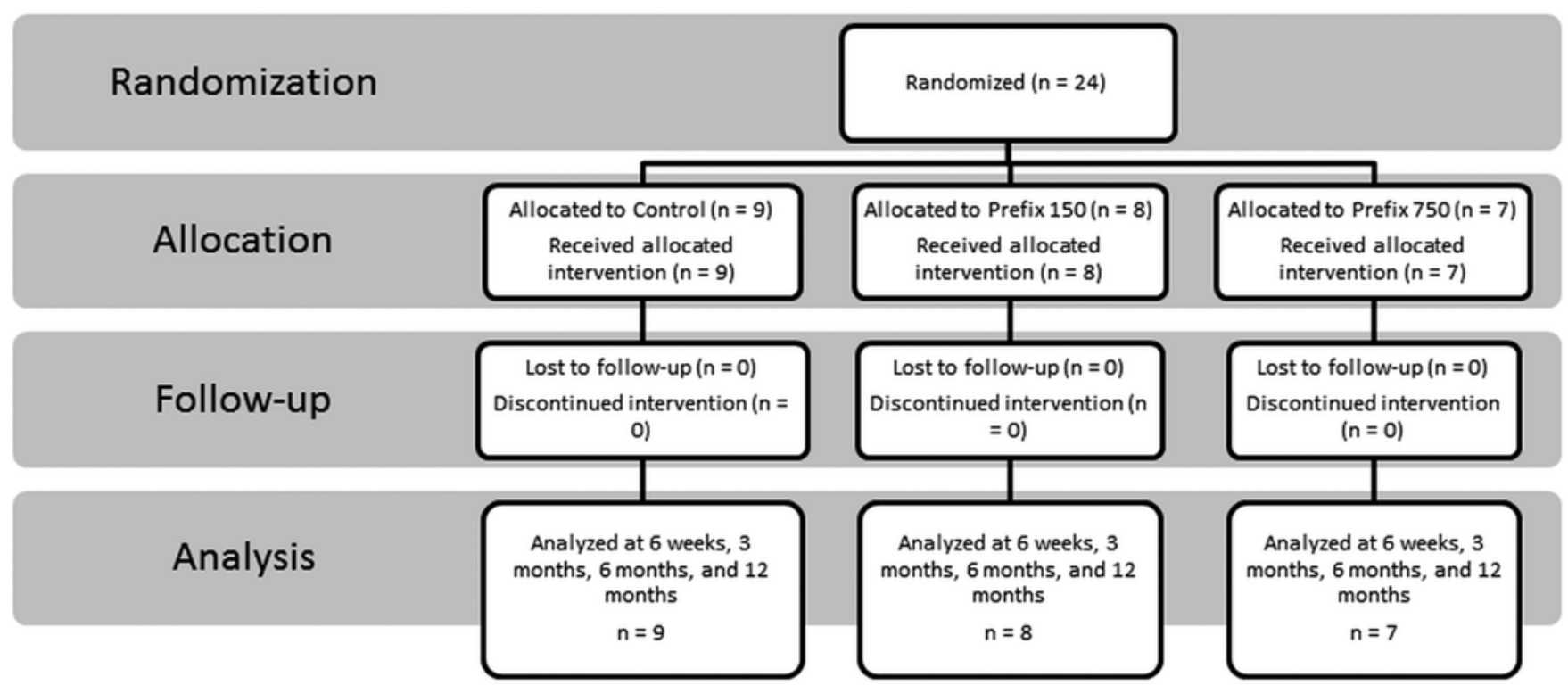

FIG. 1. Participant flow through the trial.

bic centimeters of coated granules were then mixed in a 1:1 ratio with locally derived autologous bone (from the lamina and facet joint area) giving a total of $10 \mathrm{~cm}^{3}$ of graft material to be used. For patients randomized to the control group, bone was harvested from the posterior iliac crest and was not mixed with local autograft. The appropriate graft material was then packed in and around the polyetheretherketone (PEEK) cages. The appropriately filled PEEK TLIF cages were inserted by a typical TLIF approach, which included a unilateral complete facetectomy at the diseased level to gain access to the intervertebral space. Bilateral decompression was performed when re- quired. In all groups, $1 \mathrm{~cm}^{3}$ of local autograft was packed into the decorticated contralateral facet joint. Posterior instrumentation with standard pedicle screws and rod systems was performed in all cases using the following systems: 1) Medtronic CD Horizon Legacy 5.5-mm titanium spinal set and Capstone PEEK spinal system or 2) Stryker Adaptive Vertebral PEEK Spacer (AVS) and Stryker Xia 5.5-mm titanium implant system. Perioperative data, such as blood loss, length of surgery, length of hospital stay, and complications, were recorded.

Postoperative rehabilitation plans were similar among patients and did not permit systemic treatment with non-

TABLE 1. Summary of demographic and baseline clinical characteristics*

\begin{tabular}{|c|c|c|c|c|}
\hline \multirow[b]{2}{*}{ Characteristic } & \multicolumn{3}{|c|}{ Group } & \multirow[b]{2}{*}{ p Value } \\
\hline & Control $(n=9)$ & Prefix $150(n=8)$ & Prefix $750(n=7)$ & \\
\hline Age (yrs) & $49.1 \pm 10.5$ & $44.7 \pm 11.5$ & $54.6 \pm 11.5$ & 0.25 \\
\hline Weight (lbs) & $195.1 \pm 33.6$ & $161.1 \pm 33.9$ & $196.0 \pm 26.2$ & 0.07 \\
\hline BMI & $32.4 \pm 4.0$ & $26.5 \pm 5.2$ & $28.8 \pm 3.5$ & 0.03 \\
\hline Sex & & & & 0.96 \\
\hline Male & 4 & 3 & 3 & \\
\hline Female & 5 & 5 & 4 & \\
\hline Caucasian race & 9 & 8 & 6 & 0.28 \\
\hline \multicolumn{5}{|c|}{ Baseline preop parameters } \\
\hline ODI score & $60.9 \pm 17.3$ & $49.8 \pm 9.8$ & $64.6 \pm 14.4$ & 0.13 \\
\hline \multicolumn{5}{|l|}{ VAS scores } \\
\hline Low-back pain & $8.7 \pm 1.4$ & $7.4 \pm 0.9$ & $7.9 \pm 3.2$ & 0.42 \\
\hline Right hip pain & $2.6 \pm 3.1$ & $4.2 \pm 3.0$ & $7.5 \pm 3.3$ & 0.02 \\
\hline Right leg pain & $5.7 \pm 4.1$ & $4.7 \pm 3.5$ & $6.8 \pm 3.4$ & 0.58 \\
\hline Left hip pain & $7.0 \pm 3.5$ & $5.0 \pm 2.5$ & $9.0 \pm 1.4$ & 0.03 \\
\hline Left leg pain & $7.3 \pm 3.1$ & $5.2 \pm 2.7$ & $9.0 \pm 1.5$ & 0.03 \\
\hline
\end{tabular}

* Continuous data are expressed as mean \pm SD. Values for sex and race are numbers of patients.

$\dagger$ Calculated using 1-way ANOVA. 
TABLE 2. Key inclusion and exclusion criteria for the study

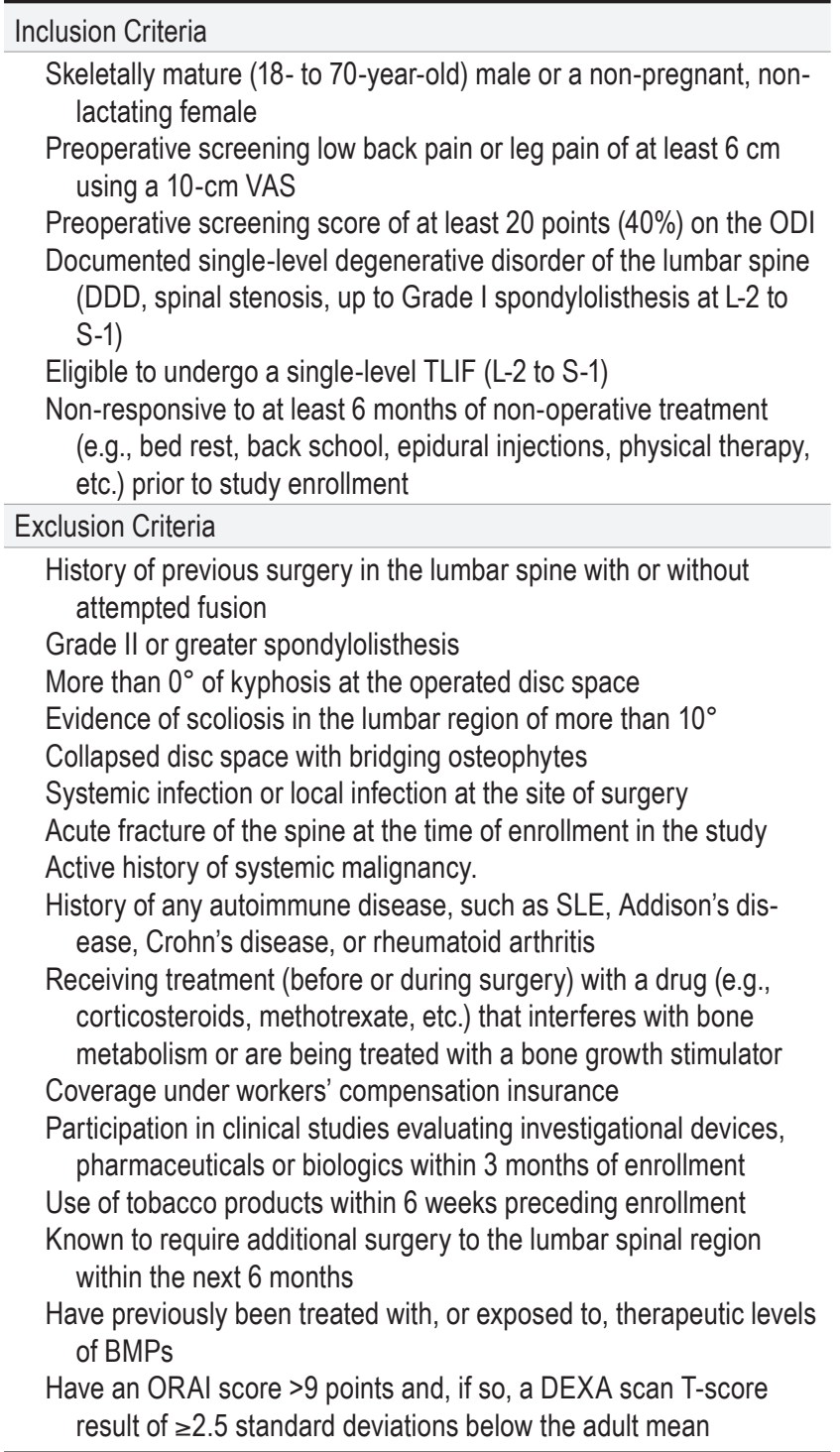

$\mathrm{BMP}=$ bone morphogenetic protein; $\mathrm{DDD}$ = degenerative disc disease; DEXA = dual-energy X-ray absorptiometry; ORAI = Osteoporosis Risk Assessment Instrument; SLE = systemic lupus erythematosus.

steroidal anti-inflammatory drugs, corticosteroids, or local injections into the index and/or adjacent vertebral levels for the first 6 months following surgery. Outcome measures included Oswestry Disability Index (ODI $)^{8}$ scores, visual analog scale (VAS) scores, and fusion outcome as assessed by CT scans (6 and 12 months postoperative) and anteroposterior, lateral, and dynamic lumbar flexion/extension plain radiographs. All radiological data were interpreted by an independent radiologist blinded to group assignment, and a quantitative software program was used to determine motion (Medical Metrics). Fusion was defined as the presence of visible bridging bone on CT scan (Figs. 2 and 3), less than $50 \%$ radiolucency around the cage, less than $5^{\circ}$ of motion, and less than 3 $\mathrm{mm}$ of translation on dynamic flexion/extension radiographs. All those criteria needed to be met for fusion to

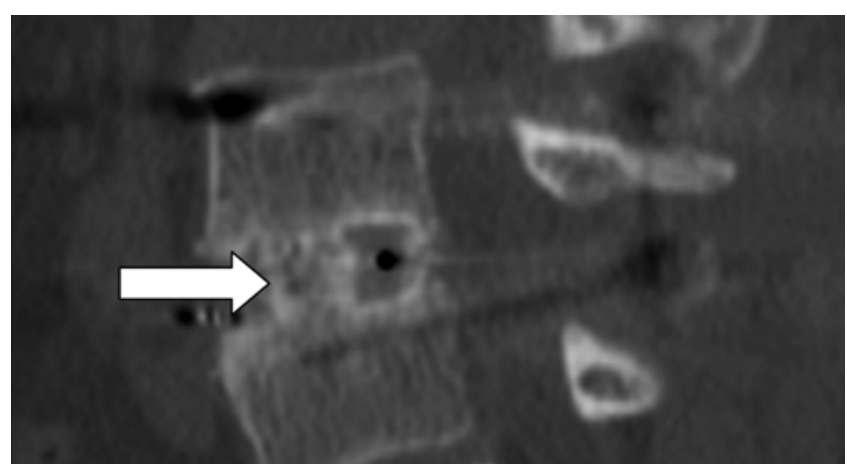

FIG. 2. CT scan showing an example of fusion at 6 months after surgery in a patient from the Prefix 750 group. The arrow indicates area of fusion between the 2 adjacent vertebrae.

be confirmed. Patients were evaluated at 6 weeks and at 3,6 , and 12 months after surgery. Neurological and radiological evaluations were completed at each visit. Routine laboratory assessment of blood samples (red blood cell count, white blood cell count, and levels of hemoglobin, sodium, chloride, potassium, calcium, inorganic phosphate, carbon dioxide, magnesium, albumin, total protein, alkaline phosphatase [ALP], gamma-glutamyl transpeptidase [GGT], aspartate aminotransferase [AST], and alanine transaminase $[\mathrm{ALT}]$ ) was performed preoperatively and again at 6 weeks and 3 months after surgery. Blood samples were also collected for immunological testing preoperatively and at 6 weeks and 3 months postoperatively and were evaluated for the potential formation of antibodies to the $\mathrm{B} 2 \mathrm{~A}$ peptide.

\section{Statistical Methods}

Statistical testing was performed using 1-way and 2-way ANOVAs for numerical results and the chi-square test for categorical data. A p value less than 0.05 was considered significant. Recognizing the potential for Type II error due to small numbers in this Phase I clinical trial, we also report results with the use of summary statistics such as mean and standard deviation.

\section{Results}

Most demographic variables were well balanced at

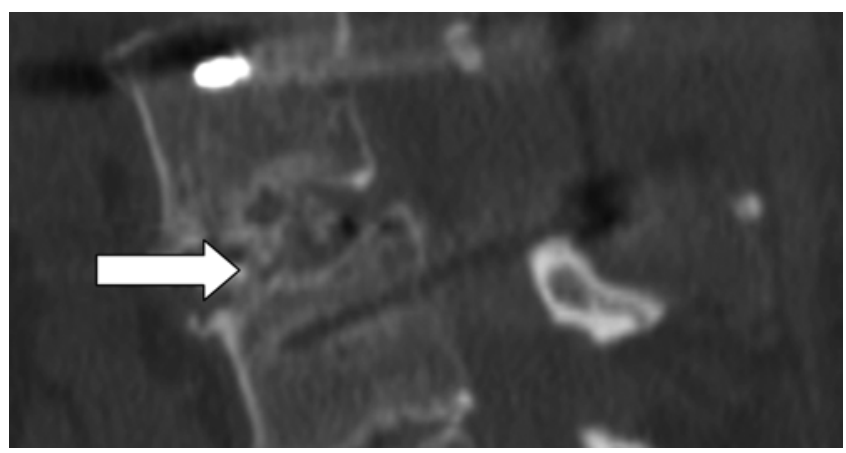

FIG. 3. CT scan showing an example of lack of fusion 6 months after surgery in a patient from the Prefix 750 group. The arrow indicates nonunion between the adjacent vertebrae. 
baseline (Table 1). ANOVA revealed a statistically significant difference in the preoperative body mass index (BMI) of the 3 groups. However, post-test analysis revealed a statistically significant difference in BMI only between the control and Prefix 150 groups.

Preoperative ODI scores and baseline VAS scores for low-back and right leg pain showed no statistically significant difference between the 3 groups (Table 1). However, ANOVA of the baseline VAS scores showed that the mean VAS scores for left leg and left hip pain in the Prefix 750 group were significantly higher than in the Prefix 150 group. The baseline VAS score for right hip pain in the Prefix 750 group was significantly higher than in the control group. There was no statistically significant difference between the baseline VAS scores in the control group and the Prefix 150 group.

The mean blood loss during surgery was higher in the control group (569 ml) than in either the Prefix 150 (364 $\mathrm{ml}$ ) or Prefix $750(314 \mathrm{ml})$ group, but this difference was not statistically significant. There was no significant difference in the length of hospital stay or the duration of surgery between the 3 groups, as shown in Table 3 .

At 6 months postsurgery (Fig. 4), the Prefix 750 group had the highest fusion rate (71.4\%), while the radiographic fusion rates in the Prefix 150 and control groups were $37.5 \%$ and $33.3 \%$, respectively; the differences were not statistically significant, however, given the small group size $(\mathrm{p}=0.27)$. By 12 months after surgery (Fig. 4), all patients in the Prefix 750 group demonstrated fusion, resulting in a radiographic fusion rate of $100 \%$, compared with a rate of $78 \%$ in the control group and only $50 \%$ in the Prefix 150 group $(\mathrm{p}=0.08)$.

The ODI scores are shown in Table 4 and Fig. 5. The Prefix 750 group had the highest mean preoperative ODI score at 64.6 (SD 13.3) compared with 60.9 (SD 16.3) for the control group and 49.8 (SD 9.1) for the Prefix 150 group ( $p>0.05)$. Six weeks postoperatively, the mean ODI score dropped to 27.7 (SD 21.1) for the Prefix 750 group, to 32.2 (SD 21.8) for the Prefix 150 group, and to only 41 (SD 11.7) for the control group ( $p>0.05$ ). However, the difference in postoperative scores diminished by 3 months after surgery, and by 12 months postoperatively, the mean ODI score was 31.1 (SD 17.6) for the Prefix 750 group, 24.4 (SD 15.7) for the control group, and 29.7 (SD 20.7) for the Prefix 150 group $(p>0.05)$. There were statistically significant within-group differences in the ODI scores for the

\section{TABLE 3. Perioperative data*}

\begin{tabular}{ccccc}
\hline & \multicolumn{3}{c}{ Group } & \\
\cline { 2 - 4 } Variable & $\begin{array}{c}\text { Control } \\
(\mathrm{n}=9)\end{array}$ & $\begin{array}{c}\text { Prefix 150 } \\
(\mathrm{n}=8)\end{array}$ & $\begin{array}{c}\text { Prefix 750 } \\
(\mathrm{n}=7)\end{array}$ & $\begin{array}{c}\mathrm{p} \\
\text { Value }\end{array}$ \\
\hline $\begin{array}{c}\text { Mean duration of } \\
\text { surgery (minutes) }\end{array}$ & $223 \pm 99$ & $203 \pm 79$ & $182 \pm 54$ & 0.61 \\
\hline Mean blood loss (ml) & $569 \pm 263$ & $364 \pm 210$ & $314 \pm 189$ & 0.07 \\
\hline $\begin{array}{c}\text { Mean length of hos- } \\
\text { pital stay (days) }\end{array}$ & $4.9 \pm 1.4$ & $4.5 \pm 2.0$ & $4.4 \pm 0.8$ & 0.78 \\
\hline
\end{tabular}

* Mean values are presented with SDs.

$\dagger$ Based on 1-way ANOVA.

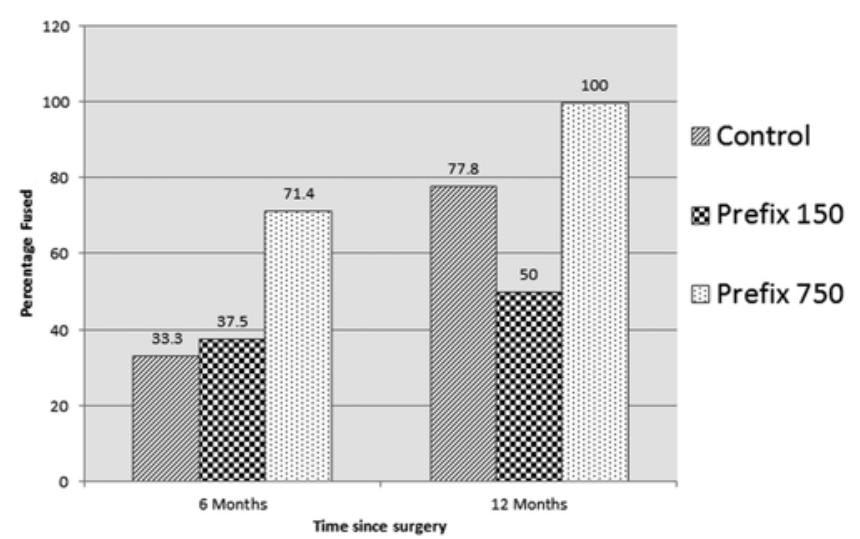

FIG. 4. Percentage of cases in which fusion was achieved in each group at 6 months and 12 months postsurgery.

control group and the Prefix 750 group from the preoperative values to all postoperative stages. However, there was no statistically significant difference in ODI values of the Prefix 150 group at the different time points. At the 12-month follow-up evaluation, $100 \%$ of patients in the Prefix 750 group had an ODI score that was at least 15 points less than their preoperative score. Similar success (100\% of patients) was seen in the control group, whereas only $62.5 \%$ of the patients in the Prefix 150 group showed this improvement of at least 15 points, with 2 patients (25\%) in the Prefix 150 group showing an increase in ODI by 12 months after surgery.

VAS scores were recorded for all patients preoperatively and then at each postoperative follow-up visit separately for low-back, left hip, left leg, right hip, and right leg pain (Table 4, Fig. 6 and 7). Compared with preoperative status, the combined VAS scores showed statistically significant improvement at all postoperative stages for all 3 groups. However, 2-way ANOVA of VAS scores showed no statistically significant difference between the 3 groups at any postoperative time point.

There were no statistically significant differences between the 3 groups with respect to complication rates (Table 5). Figure 8 shows the CT scan of a patient in the Prefix 750 group who developed adjacent-level discitis. No patients developed antibodies to the B2A peptide.

\section{Discussion}

The results of our study suggest that Prefix mixed with local autogenous bone graft is a viable alternative to iliac crest bone graft (ICBG) for achieving interbody fusion in patients undergoing the TLIF procedure. However, Prefix avoids the need for harvesting autograft from a separate site and hence avoids the complications and morbidities linked with ICBG harvesting. $.7,12-14,19,20,22,23,26,28,30,31$ Other substances used for lumbar interbody fusion have failed to show the optimal combination of efficacy and safety. Allograft has been associated with inferior fusion rates and higher graft resorption rates ${ }^{14}$ and has the potential for disease transmission and immunogenicity, ${ }^{16}$ whereas rhBMPs have been linked to a variety of complications. ${ }^{2,3,10,14,24,29}$ 
TABLE 4. Oswestry Disability Index and VAS scores at different time points

\begin{tabular}{|c|c|c|c|c|c|c|}
\hline Statistic \& Treatment Group & Pre-op & 6 Weeks & 3 Months & 6 Months & 12 Months & p Value* \\
\hline \multicolumn{7}{|l|}{ ODI score } \\
\hline \multicolumn{7}{|l|}{ Mean } \\
\hline Control & 60.9 & 41.0 & 29.8 & 24.1 & 24.4 & $<0.01(0.11)$ \\
\hline Prefix 150 & 49.8 & 32.2 & 25.5 & 26.0 & 29.7 & $0.10(0.91)$ \\
\hline Prefix 750 & 64.6 & 27.7 & 22.9 & 31.7 & 31.1 & $0.01(0.88)$ \\
\hline \multicolumn{7}{|l|}{ SD } \\
\hline Control & 16.3 & 11.7 & 14.2 & 17.9 & 15.7 & \\
\hline Prefix 150 & 9.1 & 21.8 & 20.6 & 16.1 & 20.7 & \\
\hline Prefix 750 & 13.3 & 21.2 & 20.2 & 23.8 & 17.6 & \\
\hline p Value & 0.13 & 0.40 & 0.77 & 0.76 & 0.76 & \\
\hline \multicolumn{7}{|c|}{ Combined VAS score for pain in low back \& both hips \& legs } \\
\hline \multicolumn{7}{|l|}{ Mean } \\
\hline Control & 6.3 & 1.6 & 1.3 & 1.2 & 1.4 & $<0.01(0.80)$ \\
\hline Prefix 150 & 5.3 & 1.3 & 1.4 & 1.7 & 2.2 & $<0.01(0.27)$ \\
\hline Prefix 750 & 8.0 & 1.0 & 1.2 & 2.1 & 1.9 & $<0.01(0.23)$ \\
\hline \multicolumn{7}{|l|}{ SD } \\
\hline Control & 3.7 & 2.1 & 1.8 & 1.7 & 2.2 & \\
\hline Prefix 150 & 2.8 & 1.6 & 2.3 & 2.2 & 3.1 & \\
\hline Prefix 750 & 2.7 & 1.9 & 2.5 & 3.5 & 2.7 & \\
\hline p Value & $<0.01$ & 0.38 & 0.90 & 0.26 & 0.33 & \\
\hline \multicolumn{7}{|c|}{ VAS score for low-back pain only } \\
\hline \multicolumn{7}{|l|}{ Mean } \\
\hline Control & 8.7 & 2.7 & 1.7 & 2.3 & 2.5 & $<0.01(0.81)$ \\
\hline Prefix 150 & 7.4 & 2.1 & 2.2 & 2.2 & 3.3 & $<0.01(0.82)$ \\
\hline Prefix 750 & 7.9 & 1.3 & 1.4 & 1.6 & 2.2 & $<0.01(0.92)$ \\
\hline \multicolumn{7}{|l|}{ SD } \\
\hline Control & 1.4 & 2.2 & 2.0 & 2.5 & 2.2 & \\
\hline Prefix 150 & 0.9 & 2.4 & 2.6 & 2.6 & 3.8 & \\
\hline Prefix 750 & 3.2 & 1.6 & 2.8 & 3.3 & 2.7 & \\
\hline p Value $†$ & 0.42 & 0.43 & 0.83 & 0.89 & 0.78 & \\
\hline
\end{tabular}

* Based on 1-way ANOVA for within-groups comparison of mean values across time points. Values in parentheses are the results when the preoperative values were excluded from analysis.

$\uparrow$ Based on 1-way ANOVA for between-groups comparison of mean values.

Our study demonstrated that Prefix can aid in successfully achieving lumbar interbody fusion in humans. We also demonstrated that the magnitude of this fusion is related to the concentration of Prefix used. All patients in the Prefix 750 group demonstrated fusion by 12 months compared with $77.8 \%$ in the control group and $50 \%$ in the Prefix 150 group. It seems that the Prefix 150 concentration is too low to be bioactive, and thus in this group the ceramic granules act more like uncoated ceramic granules, resulting in a fusion rate that is lower than in the control group. ${ }^{26}$ However, these differences were not statistically significant.

In this study, VAS and ODI scores followed a similar trend. Mean VAS and ODI scores were improved in all patient groups at 12 months compared with preoperative values. It is worth noting that the ODI scores for the control group were elevated (mean 41.0), although not to a statistically significant extent, compared with both Prefix groups (mean 27.7 for Prefix 750, 32.29 for Prefix 150) at 6 weeks after surgery, presumably due to the early morbidity associated with ICBG harvest. Fortunately, this effect seemed to have improved by the 12-month time point, as has been previously reported in the literature. ${ }^{23}$ Considering these early results in a small group of patients, one can surmise that Prefix 750, combined with local autogenous graft, provides an attractive alternative to ICBG, providing a safe, synthetic material that achieved fusion at least as well as ICBG autograft, while avoiding the morbidities associated with ICBG harvest. Nevertheless we acknowledge that a larger study will be necessary to fully define the safety profile of Prefix and wish to reiterate that even though we have highlighted the early differences between the 3 study groups, none of these differences were statistically significant given the small sample size.

One limitation of our study is the use of ICBG as the control group. A large number of surgeons routinely use 


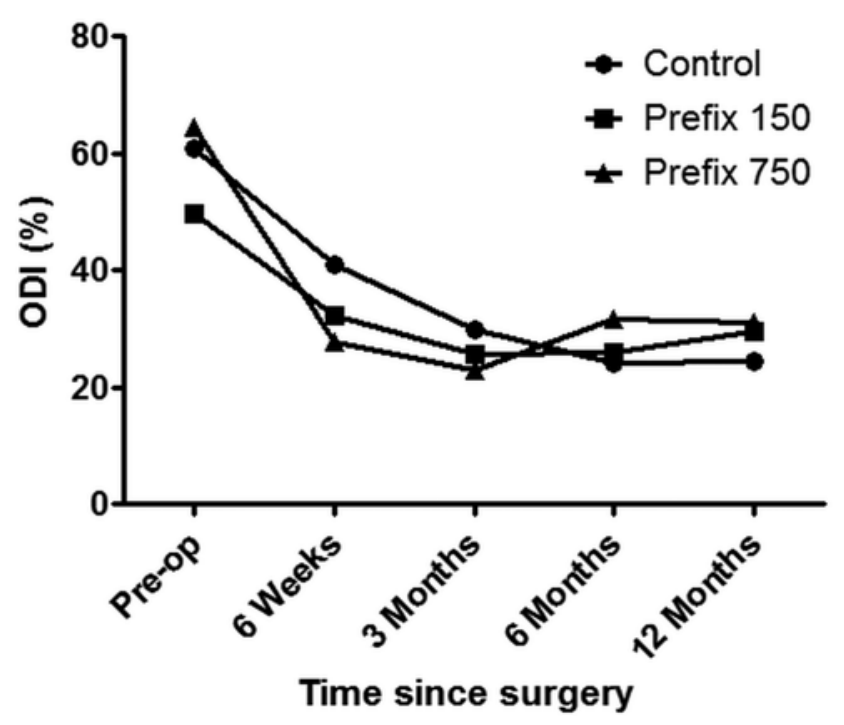

FIG. 5. Mean ODI scores.

local bone graft removed during the decompression for such procedures. Local bone has been demonstrated to be an effective source of bone for interbody fusion, with low morbidity ${ }^{17}$ and without the drawback of increased donor site morbidity. In the future, it would be interesting to compare Prefix to local autograft for interbody fusion, rather than using ICBG as a control; such a study would more clearly define any additive effects of the Prefix itself.

Safety of a product is difficult to determine in a small study, but between 3 and 4 reversible adverse events were recorded in each group. There was not a preponderance of adverse events in any one group. Of importance is the fact that there was no increase in leg pain (radiculitis) in the Prefix groups compared with the ICBG group, which has been a concerning issue with rhBMP-2. Furthermore, Prefix showed no greater evidence of causing heterotopic

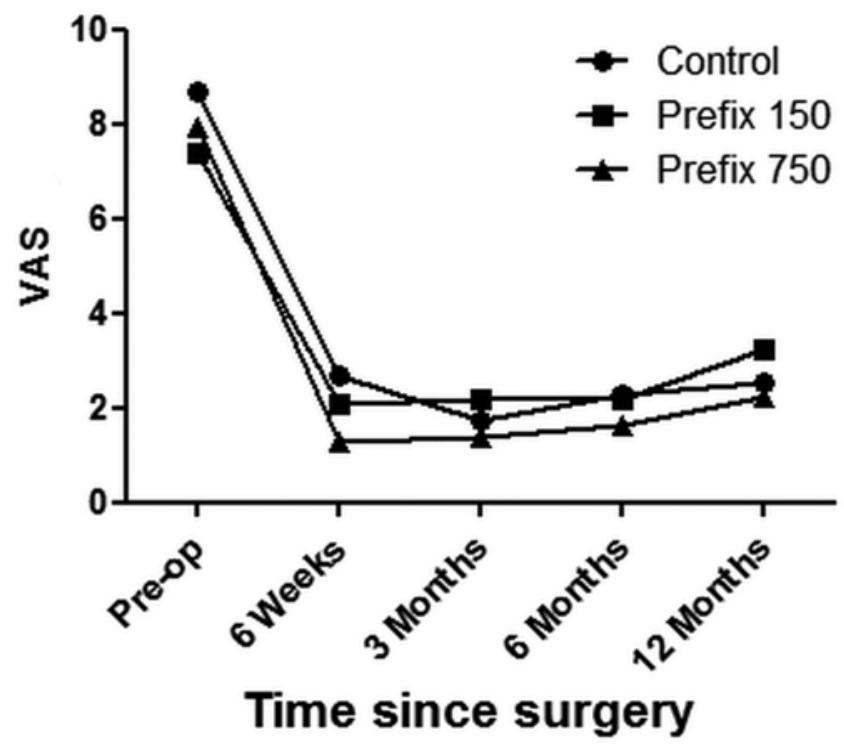

FIG. 6. Mean values for the combined VAS pain scores for both hips, both legs, and low back.

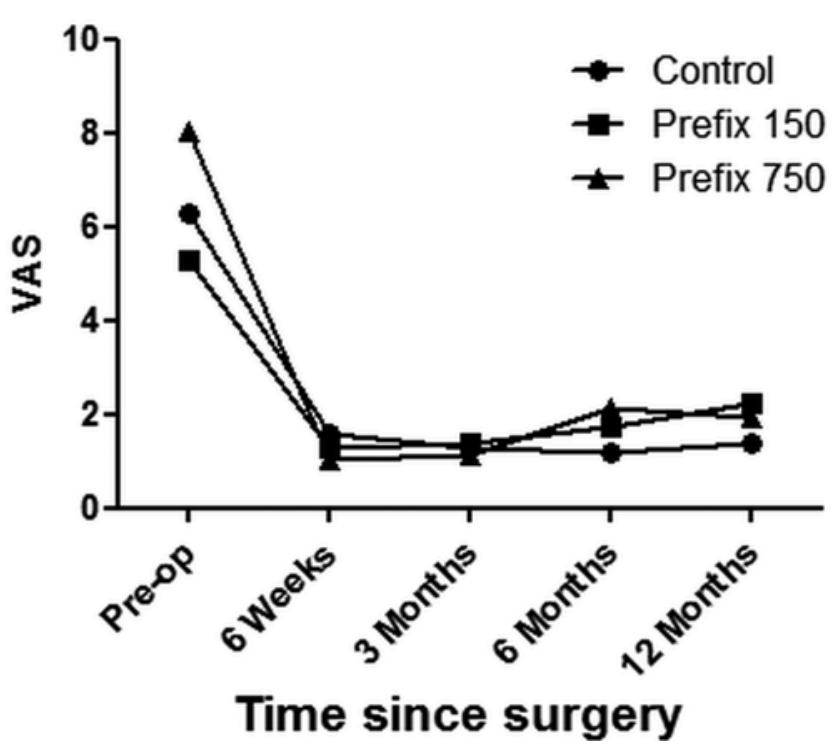

FIG. 7. Mean VAS scores for low-back pain.

TABLE 5. Postoperative adverse events in the 3 groups

\begin{tabular}{lccc}
\hline \multicolumn{1}{c}{ Adverse Event } & Control & Prefix 150 & Prefix 750 \\
\hline Myocardial Infarction & 1 & & \\
\hline Shortness of breath & & & 1 \\
\hline Transient liver enzyme increase & 1 & 1 & 1 \\
\hline Lower gastrointestinal tract bleed & 1 & & \\
\hline Delirium & 1 & & \\
\hline Superficial wound infection & & 1 & 1 \\
\hline $\begin{array}{l}\text { Adjacent-level discitis needing } \\
\text { reoperation }\end{array}$ & & & 1 \\
\hline Increased ocular pressure & & 1 & \\
\hline
\end{tabular}

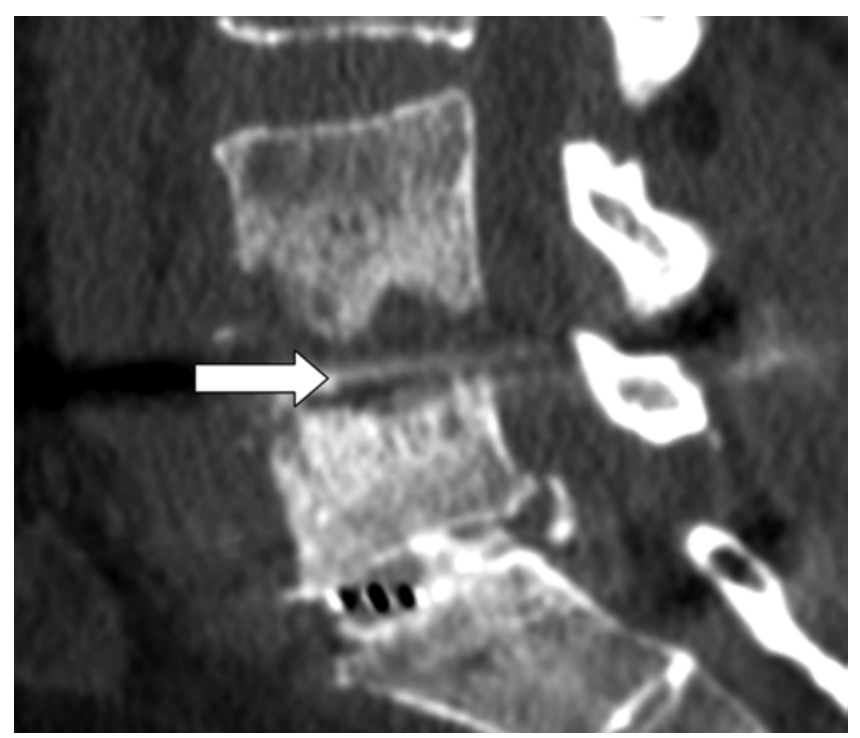

FIG. 8. CT scan demonstrating adjacent-level discitis in a patient from the Prefix 750 group. The arrow indicates the involved disc. 
bone, vertebral osteolysis, or periradicular bone formation than did ICBG. This may be related to the inability of B2A to generate bone in BMP-2-deficient environments and may avoid the documented perineural heterotopic bone formation observed with rhBMP-2, 2,3,10,14,24,29 It is acknowledged that only a large study will be able to accurately define the frequency of this relatively rare event.

\section{Conclusions}

Prefix 750 mixed with local autogenous bone graft provided a safe and effective alternative to ICBG with no lasting complication. Prefix 750 showed a numerically superior fusion rate and ODI improvement rate compared with ICBG at 12 months, although statistical significance was not reached. Prefix avoided the initial morbidity associated with ICBG while showing statistically comparable improvements in ODI and VAS scores compared with the control (ICBG) group. Prefix appears to be a promising bone graft agent and should be studied further in a larger clinical trial with a longer-term endpoint.

\section{Acknowledgments}

We would like to thank Aimee Gallant, Research Assistant, Sunnybrook Health Sciences Centre, University of Toronto, Ontario, Canada, and Dr. Darren M. Roffey, Clinical Research Associate, Ottawa Hospital Research Institute, Ottawa, Ontario, Canada.

\section{Participating Sites}

McGill University Health Centre, McGill University, Montreal, Quebec; Queen Elizabeth II Health Sciences Centre, Dalhousie University, Halifax, Nova Scotia; Foothills Medical Center, University of Calgary, Alberta; Sunnybrook Health Sciences Centre, University of Toronto, Ontario; Ottawa Hospital, University of Ottawa, Ontario, Canada.

\section{References}

1. Abbah SA, Lam CX, Ramruttun AK, Goh JC, Wong HK: Fusion performance of low-dose recombinant human bone morphogenetic protein 2 and bone marrow-derived multipotent stromal cells in biodegradable scaffolds: a comparative study in a large animal model of anterior lumbar interbody fusion. Spine (Phila Pa 1976) 36:1752-1759, 2011

2. Buttermann GR: Prospective nonrandomized comparison of an allograft with bone morphogenic protein versus an iliaccrest autograft in anterior cervical discectomy and fusion. Spine J 8:426-435, 2008

3. Carragee EJ, Hurwitz EL, Weiner BK: A critical review of recombinant human bone morphogenetic protein-2 trials in spinal surgery: emerging safety concerns and lessons learned. Spine J 11:471-491, 2011

4. Cunningham BW, Atkinson BL, Hu N, Kikkawa J, Jenis L, Bryant J, et al: Ceramic granules enhanced with B2A peptide for lumbar interbody spine fusion: an experimental study using an instrumented model in sheep. J Neurosurg Spine 10:300-307, 2009

5. De Riu G, Meloni SM, Raho MT, Gobbi R, Tullio A: Delayed iliac abscess as an unusual complication of an iliac bone graft in an orthognathic case. Int J Oral Maxillofac Surg 37:1156-1158, 2008

6. Digiovanni CW, Baumhauer J, Lin SS, Berberian WS, Flemister AS, Enna MJ, et al: Prospective, randomized, multi-center feasibility trial of rhPDGF-BB versus autolo- gous bone graft in a foot and ankle fusion model. Foot Ankle Int 32:344-354, 2011

7. Dimitriou R, Mataliotakis GI, Angoules AG, Kanakaris NK, Giannoudis PV: Complications following autologous bone graft harvesting from the iliac crest and using the RIA: a systematic review. Injury 42 (Suppl 2):S3-S15, 2011

8. Fairbank JC, Pynsent PB: The Oswestry Disability Index. Spine (Phila Pa 1976) 25:2940-2952, 2000

9. Geibel PT, Boyd DL, Slabisak V: The use of recombinant human bone morphogenic protein in posterior interbody fusions of the lumbar spine: a clinical series. J Spinal Disord Tech 22:315-320, 2009

10. Glassman SD, Howard J, Dimar J, Sweet A, Wilson G, Carreon L: Complications with recombinant human bone morphogenic protein-2 in posterolateral spine fusion: a consecutive series of 1037 cases. Spine (Phila Pa 1976) 36:1849-1854, 2011

11. Glazebrook M, Younger A, Wing K, Lalonde KA: A prospective pilot study of B2A-coated ceramic granules (Amplex) compared to autograft for ankle and hindfoot arthrodesis. Foot Ankle Int 34:1055-1063, 2013

12. Ilharreborde B, Morel E, Fitoussi F, Presedo A, Souchet P, Penneçot GF, et al: Bioactive glass as a bone substitute for spinal fusion in adolescent idiopathic scoliosis: a comparative study with iliac crest autograft. J Pediatr Orthop 28:347351, 2008

13. Ishii S, Shishido F, Miyajima M, Sakuma K, Shigihara T, Tameta T, et al: Imaging findings at the donor site after iliac crest bone harvesting. Skeletal Radiol 39:1017-1023, 2010

14. Jeong GK, Sandhu HS, Farmer J: Bone morphogenic proteins: applications in spinal surgery. HSS J 1:110-117, 2005

15. Konishi S, Nakamura H, Seki M, Nagayama R, Yamano Y: Hydroxyapatite granule graft combined with recombinant human bone morphogenic protein-2 for solid lumbar fusion. J Spinal Disord Tech 15:237-244, 2002

16. Kwon B, Jenis LG: Carrier materials for spinal fusion. Spine J 5 (6 Suppl):224S-230S, 2005

17. Lee JH, Lee JH, Park JW, Lee HS: Fusion rates of a morselized local bone graft in polyetheretherketone cages in posterior lumbar interbody fusion by quantitative analysis using consecutive three-dimensional computed tomography scans. Spine J 11:647-653, 2011

18. Lin X, Elliot JJ, Carnes DL, Fox WC, Peña LA, Campion SL, et al: Augmentation of osseous phenotypes in vivo with a synthetic peptide. J Orthop Res 25:531-539, 2007

19. Missiuna PC, Gandhi HS, Farrokhyar F, Harnett BE, Dore EM, Roberts B: Anatomically safe and minimally invasive transcrestal technique for procurement of autogenous cancellous bone graft from the mid-iliac crest. Can J Surg 54:327332, 2011

20. Myeroff C, Archdeacon M: Autogenous bone graft: donor sites and techniques. J Bone Joint Surg Am 93:2227-2236, 2011

21. Nichols TA, Sagi HC, Weber TG, Guiot BH: An alternative source of autograft bone for spinal fusion: the femur: technical case report. Neurosurgery 62:E179, 2008

22. Porchet F, Jaques B: Unusual complications at iliac crest bone graft donor site: experience with two cases. Neurosurgery 39:856-859, 1996

23. Robertson PA, Wray AC: Natural history of posterior iliac crest bone graft donation for spinal surgery: a prospective analysis of morbidity. Spine (Phila Pa 1976) 26:1473-1476, 2001

24. Rowan FE, O'Malley N, Poynton A: RhBMP-2 use in lumbar fusion surgery is associated with transient immediate postoperative leg pain. Eur Spine J 21:1331-1337, 2012

25. Schaaf H, Lendeckel S, Howaldt HP, Streckbein P: Donor site morbidity after bone harvesting from the anterior iliac crest. 


\section{Oral Surg Oral Med Oral Pathol Oral Radiol Endod 109:52-58, 2010}

26. Smucker JD, Bobst JA, Petersen EB, Nepola JV, Fredericks DC: B2A peptide on ceramic granules enhance posterolateral spinal fusion in rabbits compared with autograft. Spine (Phila Pa 1976) 33:1324-1329, 2008

27. Solchaga LA, Hee CK, Aguiar DJ, Ratliff J, Turner AS, Seim HB III, et al: Augment bone graft products compare favorably with autologous bone graft in an ovine model of lumbar interbody spine fusion. Spine (Phila Pa 1976) 37:E461E467, 2012

28. Sys J, Weyler J, Van Der Zijden T, Parizel P, Michielsen J: Platelet-rich plasma in mono-segmental posterior lumbar interbody fusion. Eur Spine J 20:1650-1657, 2011

29. Valdes MA, Thakur NA, Namdari S, Ciombor DM, Palumbo M: Recombinant bone morphogenic protein-2 in orthopaedic surgery: a review. Arch Orthop Trauma Surg 129:16511657,2009

30. Velchuru VR, Satish SG, Petri GJ, Sturzaker HG: Hernia through an iliac crest bone graft site: report of a case and review of the literature. Bull Hosp Jt Dis 63:166-168, 2006

31. Zermatten P, Wettstein M: Iliac wing fracture following graft harvesting from the anterior iliac crest: literature re- view based on a case report. Orthop Traumatol Surg Res 98:114-117, 2012

\section{Author Contributions}

Analysis and interpretation of data: Sardar. Drafting the article: Sardar. Critically revising the article: all authors. Reviewed submitted version of manuscript: all authors. Approved the final version of the manuscript on behalf of all authors: Sardar. Statistical analysis: Sardar.

\section{Supplemental Information}

Previous Presentation

Portions of this work were presented in abstract form as proceedings at the International Meeting on Advanced Spinal Techniques, Istanbul, Turkey, July 2012.

\section{Correspondence}

Zeeshan Sardar, Orthopaedic Residency Training Program, Shriners Hospital for Children-Canada, 1529 Cedar Ave., Montreal, QC H3G 1A6, Canada. email: zeeshan.sardar@mail. mcgill.ca. 